\title{
A study on the evaluation method of the characteristics of the contact point between wheel and rail
}

\author{
M. Adachi ${ }^{1} \&$ T. Shimomura ${ }^{2}$ \\ ${ }^{I}$ National Traffic Safety and Environment Laboratory, Japan \\ ${ }^{2}$ Railway Technical Research Institute, Japan
}

\begin{abstract}
It is indispensable to grasp geometrical characteristics of contact point between wheel and rail to analyze the characteristics of vehicle dynamics. However, there has not been an analytical method capable of calculating simply and easily geometrical characteristics of contact point, which is formed by actual shapes of worn tread running surface of rail and wheel tread. Therefore we have developed an analytical system by which we can calculate variations of wheel radius and contact angle each corresponding to lateral displacement of a wheel set for a combination of worn wheel tread and worn rail running surface, and finally, equivalent conicity can be obtained based on the results of calculation.

Keywords: contact angle, variations of wheel radius, equivalent conicity, contact point.
\end{abstract}

\section{Introduction}

Vehicle dynamics is greatly affected by characteristics of the creep force on the contact point between a wheel and a rail. Therefore, in order to estimate characteristics of vehicle dynamics, it is necessary to obtain exactly characteristics such as right and left wheel radius and a contact angle on the contact point based on the calculation of geometrical characteristics of the contact point between the wheel and rail.

On the other hand, when a vehicle runs on the rail, the longer distance the vehicle runs, the severer wears are evident on the wheel tread. In the same way, the more tonnage of vehicle passes severe wears on the wheel tread and the rail 
tread adversely affect the characteristics of vehicle dynamics greatly. Therefore it is essential to analyze characteristics of the contact point between the worn wheel tread and the worn rail tread.

In the past some reports, it is reported that characteristics of the contact point between the wheel and the rail tread were grasped geometrically [1-4]. However, there was not an analytical method to be able to calculate the contact point, which was formed by the actual worn shapes of the rail and wheel tread. Most of the methods by the past researches were based on assumption that the shape data of the wheel and rail could be converted to numerical formula. Therefore in this study we investigated a system by which we could calculate characteristics of the contact point between the worn wheel tread and the worn rail tread without using a function.

\section{Evaluation system of characteristics of the contact point between and the wheel and the rail}

\subsection{Outline of the evaluation system of characteristics of the contact point}

The important point of grasping characteristics of the contact point geometrically is to calculate the contact point between a right and left wheel and a rail in case a wheel set is moved in the lateral direction to the rail. Figure 1 shows a flowchart of the evaluation system of characteristics of the contact point between a wheel and a rail that we developed this time. A flow is explained as follows.

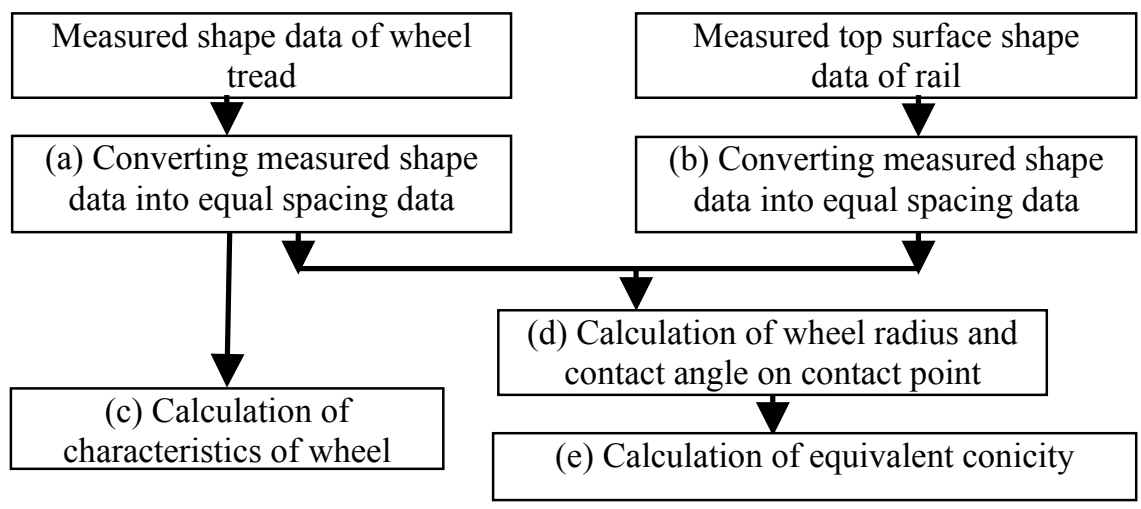

Figure 1: A flowchart of outline of the system.

(1) The measured shape data of the wheel tread we obtained by a measurement equipment are converted into the equal spacing data in the direction of wheel thickness (Figure 1 (a)).

(2) The measured top surface shape data of rail we obtained by the measurement equipment are converted into the equal spacing shape data in the direction of a cross tie in the same way as the wheel tread (Figure 1 (b)). 
(3) Characteristics of a wheel such as flange angle, flange thickness and wear depth of wheel tread are calculated based on the converted equal spacing data of the wheel tread (Figure 1 (c)).

(4) Characteristics of the contact point between a wheel and a rail are calculated. Namely the contact point is calculated based on the equal spacing data of the wheel tread and the top surface of rail, and the wheel radius and contact angle on the contact point are calculated with respect to the right and left wheel and rail (Figure $1(\mathrm{~d})$ ).

(5) Equivalent conicity is obtained based on the calculation results of the wheel radius on the contact point (Figure $1(\mathrm{e})$ ).

\subsection{Converting measured shape data into equal spacing shape data}

According to this system, the measured shape data of a wheel tread are converted into the equal spacing data in the direction of wheel thickness, and characteristics of the contact point between the wheel and the rail are calculated. Figure 2 shows an example of the measured shape data of the wheel. In the figure X-axis means the direction of the wheel thickness and Y-axis means the direction of the wheel radius. Now the measured data are not equal spacing ones in the $\mathrm{x}$ direction. Therefore the measured data must be converted into the equal spacing data in the $\mathrm{x}$ direction. Equal spacing was set at $0.1 \mathrm{~mm}$ in the $\mathrm{x}$ direction as shown in Figure 3 . The reason why the equal spacing was set at $0.1 \mathrm{~mm}$ is given below.

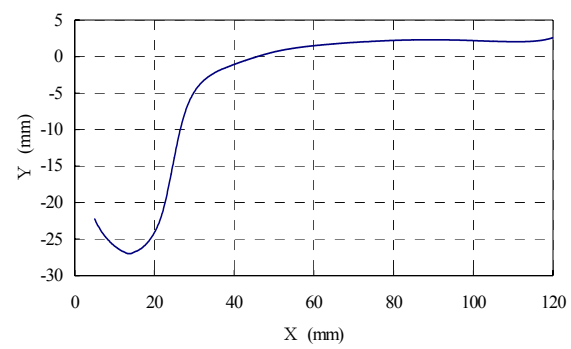

Figure 2: $\quad$ Shape data of wheel measured.

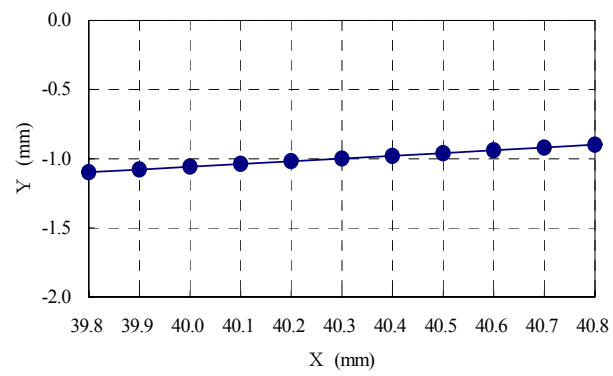

Figure 3: Shape data of wheel with linear interpolation. 
We measured the flange angle of worn wheel tread of the trial bogie equipped in the Railway Technical Research Institute. On the other hand we calculated a flange angle by using the equal spacing data of the worn wheel tread. We compared these two flange angles (how to calculate a flange angle should be referred to the section 2.3). Comparison on between the measured data and the calculated data of flange angle is shown in Figure 4. As for the calculated flange angle of equal spacing data of less than $0.2 \mathrm{~mm}$ spacing, it was almost equal to the measured value. But when the data spacing was more than $0.2 \mathrm{~mm}$, the calculation values were different from the measured values. Therefore it is obvious that the data spacing must be less than $0.2 \mathrm{~mm}$ when the data of wheel tread are to be converted into the equal spacing data. Thus in this system calculation was carried out using equal data spacing of $0.1 \mathrm{~mm}$, allowing some for $0.2 \mathrm{~mm}$. The measured data of rail were converted into the data of every 0.1 $\mathrm{mm}$ spacing in the direction of the wheel thickness just as like the wheel data, and characteristics of the contact point between the wheel and the rail were calculated based on the converted shape data of the wheel and the rail.



Figure 4: Comparison of calculation value and measured value of a flange angle.

\subsection{Characteristics of worn wheel tread}

As for characteristics of a worn wheel tread, the flange angle, flange thickness and wear depth of the wheel tread were calculated as shown in Figure 5. In the concrete, the position of the backside of the wheel is defined as $\mathrm{X}=0$ and the position of $\mathrm{Y}$ direction corresponding to $\mathrm{X}=65 \mathrm{~mm}$ is defined as $\mathrm{Y}=0$. For example, in the case of the modified arc wheel profile of a meter gauged train, the profile of the wheel tread between a point at $\mathrm{Y}=-12 \mathrm{~mm}$ and that at $\mathrm{Y}=-18$ $\mathrm{mm}$ was calculated as an approximate straight line with the least-squares method, and an angle of the line was defined as a flange angle. Flange thickness was defined as the value of $X$ corresponding to $Y=-10 \mathrm{~mm}$. Wear depth was defined as difference between the value of $Y$ corresponding to $X=65 \mathrm{~mm}$ of worn wheel tread and the value of $Y$ corresponding to $X=65 \mathrm{~mm}$ of standard design shape of wheel. Characteristics of worn wheel tread, mentioned above, were calculated for right and left wheel respectively. 


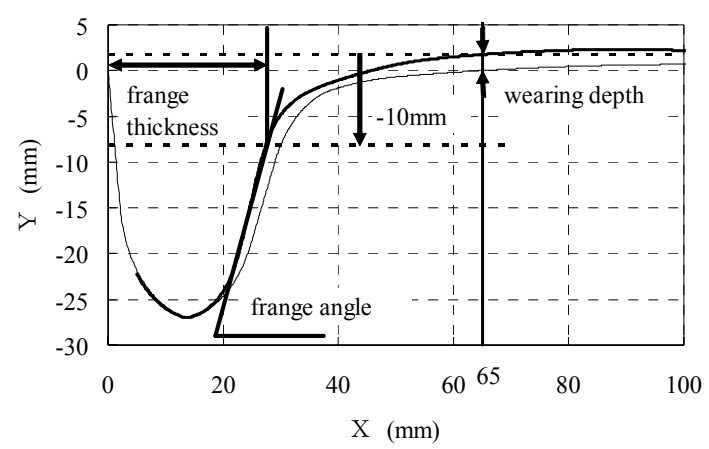

Figure 5: A figure to calculate characteristics of wheel.

\subsection{Calculation of characteristics of the contact point between wheel and rail}

\subsubsection{Setting wheel and rail}

Wheels and rails must be set in a predetermined position to analyze characteristics of the contact point between a wheel and a rail. Therefore, the shape data of both right and left wheel and those of rails which were treated individually were set in the absolute space considering the rail gauge and the angle of tie plate. At first right and left wheels were set with a defined space between them, constituting a wheel set, and right and left rails were set with a defined space between them in the same way. Then each wheel was set in a neutral position on each rail. Next each wheel was moved in parallel in the direction of top surface of rail by predetermined distance. The direction of Xaxis means the direction along a wheel set (the direction of a wheel thickness) and the direction of $\mathrm{Y}$-axis means the height direction of a wheel.

\subsubsection{Rolling of wheel set}

After wheels and rails were set in a predetermined position, each right and left wheel was moved in parallel by the distance of $\mathrm{Xw}$ in the direction of $\mathrm{X}$-axis. Then the distance between each wheel and each rail in the $\mathrm{Y}$ direction were calculated. Each right and left wheel are considered to come in contact with each right and left rail under the consideration that a wheel set had some inclination. So keeping relative position of right and left wheel as a wheel set, a wheel set was rolled around the horizontal axis (in the direction of train running) at the center of wheel set, and the distance of Y direction between each wheel and rail was calculated.

\subsubsection{Calculation of contact point}

For the right and the left wheel each, the distance between the wheel and the rail in the Y direction, HL (between the left wheel and the left rail) and HR (between the right wheel and the right rail) were calculated. The distance between the wheel and the rail was defined as the minimum of difference of height between 
the wheel tread and the top surface of rail as shown in Figure 6. HL and HR were calculated and their difference was obtained. If their difference was not more than $1 / 10000 \mathrm{~mm}$ when the roll angle of wheel set, $\phi \mathrm{w}$, was changed by dichotomy, HL and HR were assumed to be equal to each other. In other words it was assumed that wheels came in contact with rails with the roll angle $\phi \mathrm{w}$.

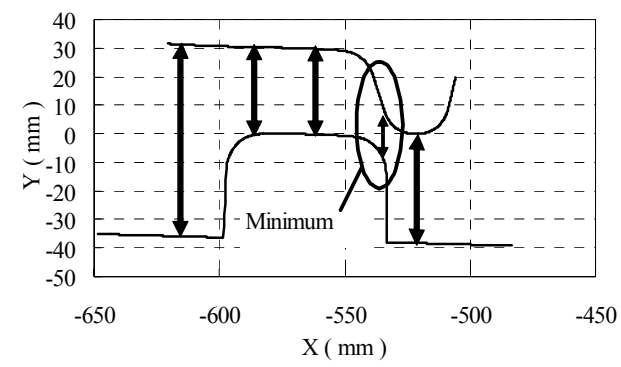

Figure 6: Calculation minimum of distance between wheel and rail.

After the contact point was decided, the wheel radius and the contact angle on the contact point were calculated for the right and left wheel each as shown in Figure 7. Contact angle was defined as an inclination of a tangent line at the contact point. After the lateral displacement of the wheel set on the rail, Xw, was changed by every $0.1 \mathrm{~mm}$ in the range from $-\mathrm{Xwmin}$ (minimum) to $+\mathrm{Xwmax}$ (maximum), the contact point, the wheel radius and the contact angle corresponding to $\mathrm{Xw}$ were calculated.

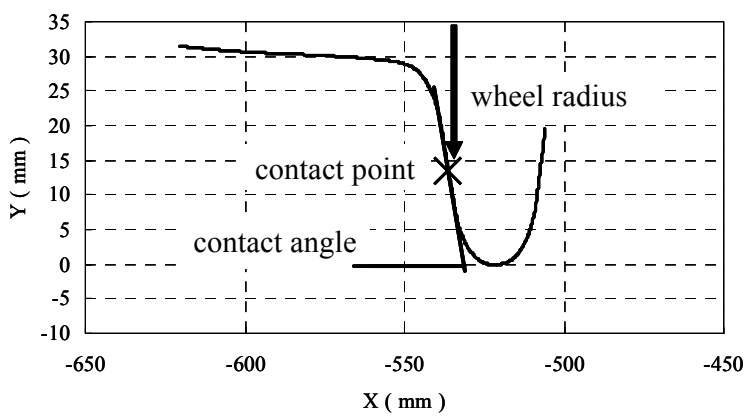

Figure 7: Calculation of characteristics in contact point between wheel and rail.

\subsubsection{Calculation of equivalent conisity}

A simple rolling wave length (rate of slip $=0$ ) was calculated by using the time career simulation, based on the wheel radius calculated above, the equations (1) and (2), for the case a wheel set was given the initial lateral displacement (for 
example $3 \mathrm{~mm}$ ) as shown in Figure 8. By substituting calculated wave length into the equation (3), equivalent conicity was obtained.

$$
\begin{gathered}
d X_{W} / d t=V \cdot \varphi_{W} \\
d \varphi_{W} / d t=\left\{\left(r_{L}-r_{R}\right) / r_{0}\right\} \cdot V / 2 b \\
S_{1}=2 \pi\left(b r_{0} / \lambda_{e}\right)^{1 / 2}
\end{gathered}
$$

where $X_{W}$ : lateral displacement of a wheel set

$V:$ speed

$\varphi_{W}$ : yaw angle of a wheel set

$S_{1}$ : wave length of a wheel set hunting

$r_{0}$ :wheel radius in neutral position

$r_{L}:$ radius of left wheel

$r_{R}:$ radius of right wheel

$\lambda_{e}:$ equivalent conicity

$2 b:$ distance between right and left contact points



Figure 8: $\quad$ Figure showing calculation of wavelength of a wheel set hunting.

\section{Analysis by evaluation system of characteristics of contact point}

\subsection{Contact state diagram}

The system can calculate characteristics of the contact point between a wheel and a rail. In order to confirm whether calculation of the contact point was right or not, an example of contact state diagram of a wheel and a rail is shown in Figure 9. Rail was fixed at a predetermined position, and displacement of a wheel set in the lateral direction to the rail, Xw, were set at $0,5,10$ and $15 \mathrm{~mm}$. The left side of Figure 10 shows the contact state diagram of the left wheel and the left rail, and the right side of the figure shows that of right wheel and the right rail. It is clear from the Figure 10, that the system can properly calculate the contact point between a wheel and a rail, and the calculation result is correct.

\subsection{Variations of wheel radius and contact angle}

Figure 10 shows the relation between the lateral displacement of a wheel set on the rail and variations of wheel radius, which were obtained by calculation of the contact point. Variations of the wheel radius compared with radius of design 

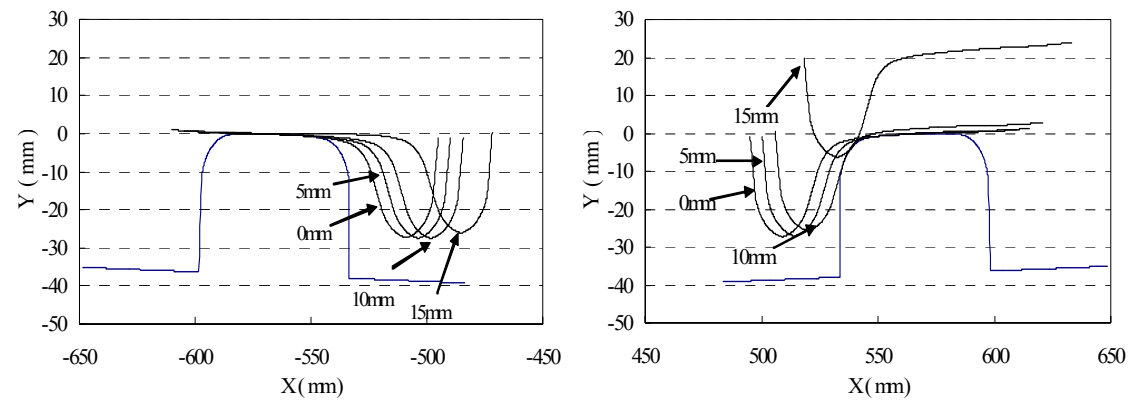

Figure 9: $\quad$ Contact diagram of wheel and rail.

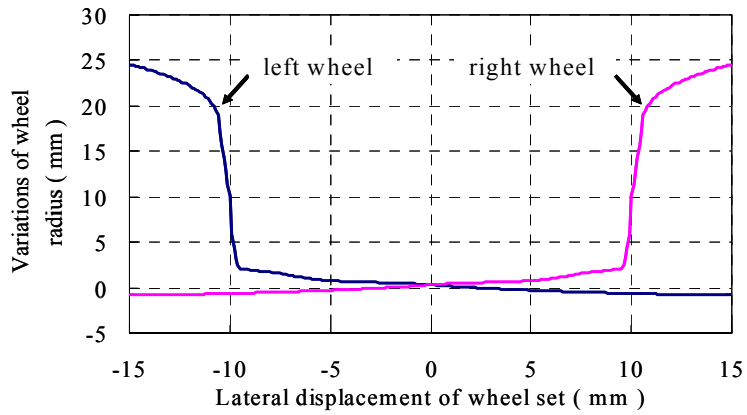

Figure 10: Relation between lateral displacement of wheel set and variations of wheel radius.

shape wheel (wheel radius in the position of $65 \mathrm{~mm}$ from backside of wheel: standard value) were calculated for each right and left wheel. Figure 11 shows relation between the lateral displacement of a wheel on the rail and the contact angle.

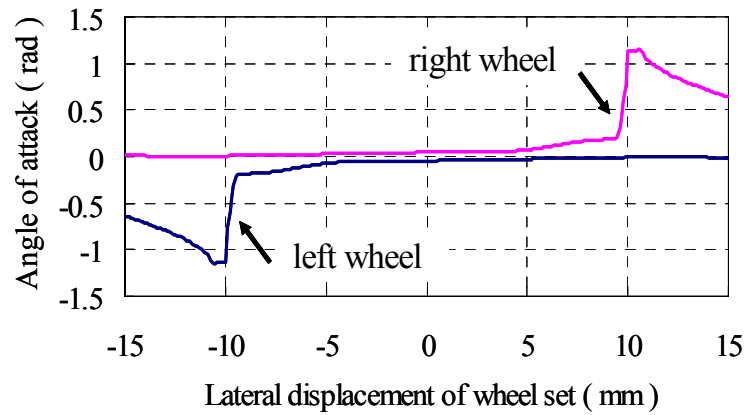

Figure 11: Relation between lateral displacement and contact angle. 


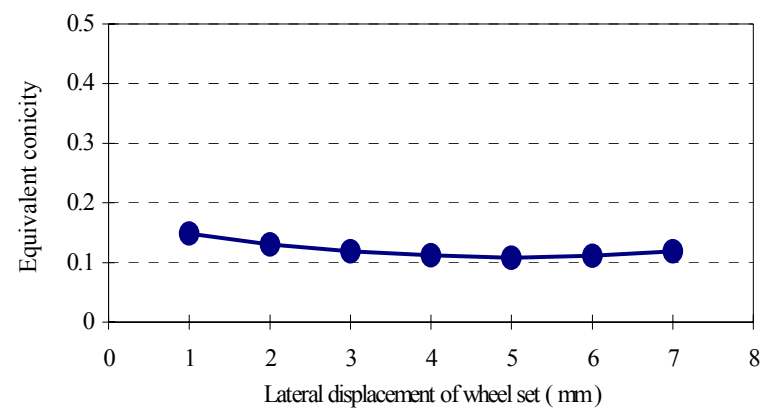

Figure 12: Relation between lateral displacement and equivalent conicity.

\subsection{Equivalent conicity}

Figure 13 shows relation between initial lateral displacement of a wheel set on the rail and equivalent conicity, which was obtained by using results of analysis of relation between the lateral displacement of a wheel set on the rail and variations of the wheel radius. It is clear from the figure that equivalent conicity varies corresponding to initial lateral displacement of the wheel set. Therefore in future we must study initial lateral displacement of wheel set which can derive realistically possible equivalent conicity.



Figure 13: Calculation result by the three-dimensional contact analysis.

\section{Verification of analysis results}

\subsection{Verification of calculation results of design shape tread}

The calculation program of a three-dimensional contact state that was reported before [4] was used to analyze characteristics of the contact point between design wheel shape and design rail shape. Now we compared the calculation result by the calculation program of a three-dimensional contact with that by our developed system. Combinations of wheel and rail used for calculation were modified arc wheel profile and $60 \mathrm{~kg}$ rail, modified arc wheel profile and $50 \mathrm{kgN}$ 
rail, arc wheel profile and $60 \mathrm{~kg}$ rail. For these combinations of wheel and rail the relation between lateral displacement of wheel set on the rail and variations of wheel radius was calculated. The results of calculation are shown in Figure 13 and Figure 14. Calculation results by the developed system were well similar to those by the calculation program of a three-dimensional contact. Therefore we could confirm that the developed system was effectiveness for the combinations of design wheel shape and design rail shape.



Figure 14: Calculation result by the development system.

\subsection{Verification of calculation results of wear tread}

We analyzed characteristics of the contact point by using the worn tread and the worn rail that were kept in RTRI, and calculated the relation between lateral displacement of the wheel set on the rail and variations of the wheel radius as shown in Figure 15. Further we calculated the contact points by CAD with the

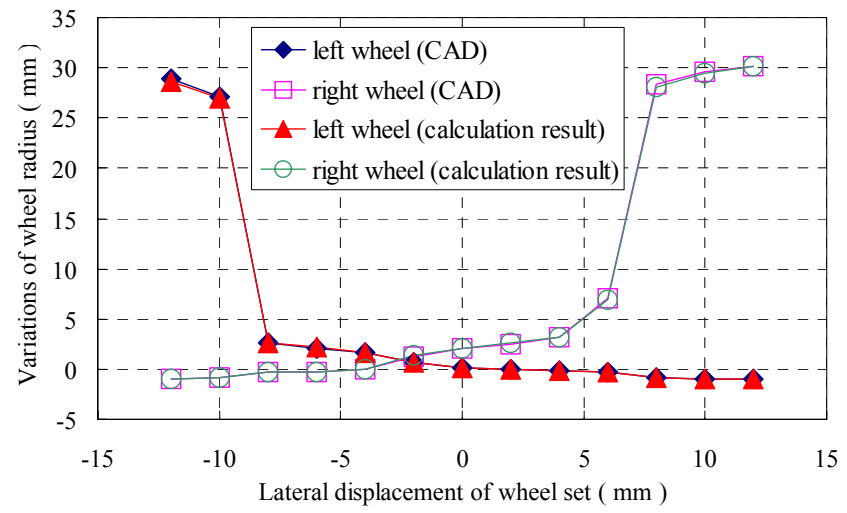

Figure 15: Comparison of calculation result with that by CAD wheel wear tread and rail wear tread. 
use of the same combination of wheel and rail. Thus we obtained relation between lateral displacement of wheel set on the rail and variations of wheel radius as shown in the same figure. Wheel set was moved by every $2.0 \mathrm{~mm}$ in the lateral direction on the rail. Calculation results by the developed system were well similar to those of analysis by CAD. Therefore we could confirm that the developed system was effectiveness for combinations of worn wheel tread and worn rail top surface likewise.

\section{Conclusion}

The measured data of wheel tread shape and the measured top shape data of rail surface with measurement equipment were converted into the equal spacing data of $0.1 \mathrm{~mm}$ in the direction of wheel thickness. The new system developed by us could easily and simply calculate characteristics of the contact point between wheel and rail by using converted data of wheel shape and rail shape with a commercial personal computer. Thus by this system we could obtain the contact point between the wheel and the rail, the wheel radius and the contact angle each corresponding to the lateral displacement of wheel set on the rail and equivalent conicity.

\section{References}

[1] Fujioka, T. \& Iguchi, M., Fundamental Study on Vehicle Dynamics, (1st Report, Contact Geometry), Transactions of the Japan Society of Mechanical Engineers C, 49-444(1983), 1354-1361

[2] Fujioka, T. \& Mitsuhira, G., Contact Geometry Between a Wheelset and Track (2nd Report, Three Dimensional Contact Geometry for Measured Worn Profiles), Transactions of the Japan Society of Mechanical Engineers C, 65-629(1991), 255-260

[3] Fujioka, T. \& Mitsuhira, G., Contact Geometry Between a Wheelset and Track (Calculation Algorithms and Contact Geometry on Switch Track), Transactions of the Japan Society of Mechanical Engineers C, 65629(1999), 249-254

[4] Sato, E., Study on Dynamics of an Independent Wheel Bogie with Steering Device for Railway Vehicle, RTRI REPORT, Special No.37 (2000),17-56 